\begin{tabular}{|c|l|}
\hline Title & Bulk-boundary correspondence for chiral symmetric quantum walks \\
\hline Author(s) & A sboth, Janos K.; Obuse, Hideaki \\
\hline Citation & $\begin{array}{l}\text { Physical review b, 88(12), 121406 } \\
\text { https://doi.org/10.1103/PhysRevB.88.121406 }\end{array}$ \\
\hline Issue Date & 2013-09-17 \\
\hline Doc URL & http://hdl.handle.net/2115/53443 \\
\hline Rights & ○2013A merican Physical Society \\
\hline Type & article \\
\hline File Information & e121406.pdf \\
\hline
\end{tabular}

Instructions for use 


\title{
Bulk-boundary correspondence for chiral symmetric quantum walks
}

\author{
János K. Asbóth ${ }^{1}$ and Hideaki Obuse ${ }^{2}$ \\ ${ }^{1}$ Institute for Solid State Physics and Optics, Wigner Research Centre, Hungarian Academy of Sciences, \\ H-1525 Budapest P.O. Box 49, Hungary \\ ${ }^{2}$ Department of Applied Physics, Hokkaido University, Sapporo 060-8628, Japan
}

(Received 5 March 2013; published 17 September 2013)

\begin{abstract}
Discrete-time quantum walks (DTQW) have topological phases that are richer than those of time-independent lattice Hamiltonians. Even the basic symmetries, on which the standard classification of topological insulators hinges, have not yet been properly defined for quantum walks. We introduce the key tool of time frames, i.e., we describe a DTQW by the ensemble of time-shifted unitary time-step operators belonging to the walk. This gives us a way to consistently define chiral symmetry (CS) for DTQW's. We show that CS can be ensured by using an "inversion symmetric" pulse sequence. For one-dimensional DTQW's with CS, we identify the bulk $\mathbb{Z} \times \mathbb{Z}$ topological invariant that controls the number of topologically protected 0 and $\pi$ energy edge states at the interfaces between different domains, and give simple formulas for these invariants. We illustrate this bulk-boundary correspondence for DTQW's on the example of the "4-step quantum walk," where tuning CS and particle-hole symmetry realizes edge states in various symmetry classes.
\end{abstract}

DOI: 10.1103/PhysRevB.88.121406

PACS number(s): 03.65.Vf, 03.67.-a, 05.30.Rt, 05.40.Fb

The realization that band insulators can have nontrivial topological properties that determine the low-energy physics at their boundary has been a rich source of new physics in the last decade. The general theory of topological insulators and superconductors ${ }^{1,2}$ classifies gapped Hamiltonians according to their dimension and their symmetries. ${ }^{3}$ As very few real-life materials are topological insulators, there is a strong push to develop model systems, "artificial materials," that simulate topological phases. ${ }^{4}$ One of the promising approaches is to use discrete-time quantum walks (DTQW) ${ }^{5-8}$ which can simulate topological insulators from all symmetry classes in 1D and 2D. ${ }^{9-11}$

DTQW's with particle-hole symmetry (PHS) go beyond simulating topological insulating Hamiltonians: they have topological phases with no counterpart in standard solid-state setups. In 1D DTQW's with PHS, edge states, "Majorana modes" can have two protected quasienergies: $\varepsilon=0$ or $\pi$ (time is measured in units of the time step and $\hbar=1$ ). Building on the results for periodically driven systems, ${ }^{12}$ one of us has defined the corresponding $\mathbb{Z}_{2} \times \mathbb{Z}_{2}$ topological invariant. ${ }^{13}$ Both 0 and $\pi$ energy Majorana edge states have been experimentally observed in a quantum walk. ${ }^{14}$

The situation of chiral symmetry (CS) of DTQW's is much less clear. Even for the simplest one-dimensional DTQW, it is disputed whether it even has $\mathrm{CS}^{9}$ or not. ${ }^{13}$ Although it is expected that CS should imply a $\mathbb{Z} \times \mathbb{Z}$ bulk topological invariant, this has not yet been found for DTQW's. As opposed to the case of PHS, there is also not much to draw on from periodically driven systems. What DTQW's have CS? How can the bulk "winding number" be expressed for DTQW's with CS? These are the problems we tackle in this Rapid Communication.

A DTQW concerns the dynamics of a particle, "walker," whose wave function is given by a vector, $|\Psi\rangle=$ $\sum_{x=1}^{N} \sum_{s=-1,1} \Psi(x, s)|x, s\rangle$. Here, $x=1, \ldots, N$ is the discrete position, and $s= \pm 1$ indexes the two orthogonal internal states of the walker, the "coin eigenstates," which we also refer to as "spin." The dynamics, instead of given by a time-independent Hamiltonian, is realized using a periodic sequence of alternating "step" and "coin rotation" operations.

The step operations are translations of the particle by one lattice site depending on the value of the "coin," the $z$ component of its spin. These are described by unitary operators $S_{s}$, where $s$ is either + or - , and

$$
S_{ \pm}=\sum_{x=1}^{N}(|x \pm 1\rangle\langle x|\otimes| \pm 1\rangle\langle \pm 1|+| x\rangle\langle x|\otimes| \mp 1\rangle\langle\mp 1|) .
$$

For simplicity, we take periodic boundary conditions.

Between each two steps, a site-dependent local "coin rotation" $R_{j}$ on the walker's internal state is performed. We consider

$$
\begin{gathered}
R_{j}=\sum_{x=1}^{N}|x\rangle\langle x| \otimes R\left(\chi_{j}(x), \theta_{j}(x)\right), \\
R(\chi, \theta)=\left(\begin{array}{cc}
\cos \theta-i \sin \theta \sin \chi & -\sin \theta \cos \chi \\
\sin \theta \cos \chi & \cos \theta+i \sin \theta \sin \chi
\end{array}\right) \\
=\exp \left[-i \theta\left(\cos (\chi) \sigma_{y}+\sin (\chi) \sigma_{z}\right)\right] .
\end{gathered}
$$

This allows breaking PHS via the angle $\chi \cdot{ }^{9}$ Details of how the local operations $R_{j}$ are performed do not influence the DTQW, all the information about them is summarized in the corresponding unitaries $R_{j}$.

One period of the DTQW is defined by $|\Psi(t+1)\rangle=$ $U_{0}|\Psi(t)\rangle$, for $t \in \mathbb{Z}$. Here, the unitary time-step (Floquet) operator is composed of $2 M$ successive pulses,

$$
U_{0}=S_{M} R_{M} S_{M-1} R_{M-1} \ldots S_{1} R_{1} .
$$

A period has to include an equal number of $S_{+}$and $S_{-}$pulses, otherwise the time-step operator has quasienergy winding, ${ }^{15}$ and cannot have gaps. Thus $M$ is even. We take each pulse to have a duration $1 / 2 M$, without losing generality.

We can also shift the starting time of the period by $T$, giving the dynamics as $|\Psi(t+1+T)\rangle=U_{T}|\Psi(t+T)\rangle$, for any $t \in$ $\mathbb{Z}$. We refer to this shift, illustrated in Fig. 1 as going into the "time frame" $T$. The starting time $T$ has to be during a rotation, 


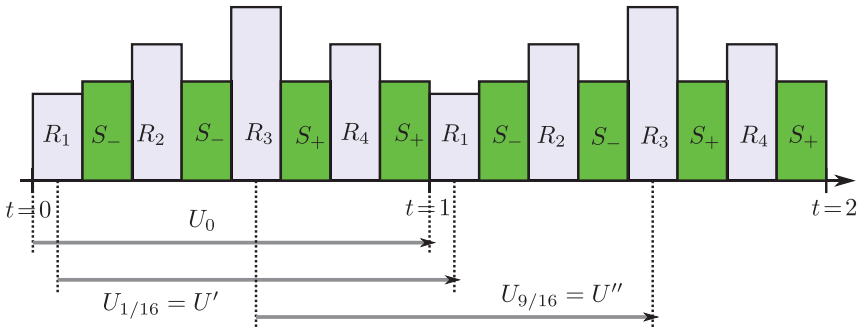

FIG. 1. (Color online) A DTQW is defined by a periodic sequence of pulses: site-dependent spin rotations $R_{j}$, Eq. (2), and spindependent translations $S_{+}$and $S_{-}$, Eq. (1). The unitary time-step operator $U_{0}$ corresponds to a complete period, as in Eq. (5). The same quantum walk can also be described in different "time frames," i.e., by time-shifted time-step operators $U_{T}$ as in Eq. (6). Two examples are shown, with $U^{\prime}$ defined by $T=1 / 16$ and $U^{\prime \prime}$ by $T=9 / 16$.

since performing only part of a shift operation would leave the walker between sites, and its description would necessitate an increased Hilbert space. This restricts $T$ to $T=(l-1) / M+$ $y /(2 M)$, for $1 \leqslant l<M$, and $0 \leqslant y<1$. The Floquet operator in the time frame $T$ reads

$$
U_{T}=R_{M+l}^{y} S_{M+l-1} R_{M+l-1} \ldots S_{l+1} R_{l+1} S_{l} R_{l}^{1-y},
$$

where we define $R_{j}^{y} \equiv \sum_{x}|x\rangle\langle x| \otimes R\left(\chi_{j}(x), y \theta_{j}(x)\right)$. Note that $U_{T}$ is a unitary transform of $U_{0}$.

A DTQW can be seen as a stroboscopic simulator of an effective Hamiltonian $H_{\mathrm{eff}, T}$. The effective Hamiltonian is associated to the Floquet operator by

$$
U_{T} \equiv e^{-i H_{\mathrm{eff}, T}} .
$$

The effective Hamiltonian is uniquely defined by this equation if we restrict its eigenvalues, the quasienergies, to an "energy Brillouin zone," $-\pi<\varepsilon \leqslant \pi$. This is completely analogous to the restriction of the quasimomentum to the first Brillouin zone.

Previous work on CS in DTQWs has focused on a single time frame, whether $H_{\text {eff, } 0}$ has CS, and identifying the associated topological invariant. Our crucial insight is that it is important to widen the scope: a DTQW has $C S$, if there is a time frame where its effective Hamiltonian has $C S$, in other words, if a time $T$ and a unitary operator $\Gamma$ acting on the coin space can be found, with $\Gamma^{2}=1$, that $\Gamma U_{T} \Gamma=U_{T}^{-1}$.

A sufficient condition for a DTQW to have CS represented by $\Gamma=\sigma_{x}$ is that the sequence of operations defining the walk has an "inversion point." By this, we mean that there is an $l$, with which for every $j$ :

$$
\begin{gathered}
R_{l-j}=R_{l+j}, \\
S_{l-j}=S_{+} \leftrightarrow S_{l+j+1}=S_{-} .
\end{gathered}
$$

We choose $\Gamma=\sigma_{x}$ for two reasons. First,

$$
\sigma_{x} S_{-} \sigma_{x}=S_{+}^{-1}
$$

whereby also $\sigma_{x} S_{+} \sigma_{x}=S_{-}^{-1}$. Second, since the local unitaries are rotations $R_{j}$ about axes that have no $x$ component,

$$
\sigma_{x} R(\chi, \theta) \sigma_{x}=R(\chi, \theta)^{-1}=R(\chi,-\theta) .
$$

Consider the sequences of $M$ operations just after and just before the middle of the "inversion point,"

$$
\begin{aligned}
& F=R_{l+M / 2}^{1 / 2} S_{l-1+M / 2} R_{l-1+M / 2} \ldots R_{l+1} S_{l} R_{l}^{1 / 2}, \\
& G=R_{l}^{1 / 2} S_{l-1} R_{l-1} \ldots R_{l+1-M / 2} S_{l-M / 2} R_{l-M / 2}^{1 / 2} .
\end{aligned}
$$

These give us two Floquet operators for the walk:

$$
U^{\prime}=F G, \quad U^{\prime \prime}=G F,
$$

as shown in Fig. 1. Using relations (10) and (11), we have that time reversal can be done during a period, $\Gamma F \Gamma G=1$, whereby $G=\Gamma F^{-1} \Gamma$. From this it is straightforward to show that both $U^{\prime}$ and $U^{\prime \prime}$ are chiral symmetric. Thus, "inversion symmetry" of the DTQW sequence in the sense of Eq. (9) gives two inequivalent "CS time frames:" time frames where the effective Hamiltonian of the DTQW has CS.

CS allows a definition of sublattices, via the projection operators $\Pi_{A}=(1+\Gamma) / 2, \Pi_{B}=(1-\Gamma) / 2$. Eigenstates of $H_{\mathrm{eff}}^{\prime}$ with quasienergy $\varepsilon \neq 0, \pi$ can be chosen to have equal support on both sublattices. Stationary states with quasienergies 0 or $\pi$, however, can be chosen to be on a single sublattice in a time frame with a CS Floquet operator, $U^{\prime}$ (their wave functions in this time frame are eigenstates of $\Gamma$ ).

We now proceed to derive the bulk-boundary correspondence for DTQW with CS. We consider an inhomogeneous DTQW with CS, consisting of a translationally invariant " $L$ " bulk at $1 \ll x \ll d$ and an " $R$ " bulk at $d \ll x \ll N$. There are (smooth or sharp) boundaries between the two bulks around $x \approx d$ and 1 . In the time frame where the Floquet operator $U^{\prime}$ has CS, the two bulks have effective Hamiltonians $H_{\mathrm{eff}, L}^{\prime}$ and $H_{\text {eff, } R}^{\prime}$. We assume both bulk Hamiltonians have gaps around $\varepsilon=0$ and $\pi$. Therefore, if stationary states with quasienergies $\varepsilon=0$ or $\pi$ exist, they must have wave functions confined to the edges, exponentially decaying towards the bulks. The number of edge states at the edge around $x \approx d$ on sublattice $A(B)$ is $m_{A}^{\prime}\left(m_{B}^{\prime}\right)$. These can further be written as

$$
m_{A}^{\prime}=m_{A, 0}^{\prime}+m_{A, \pi}^{\prime}, \quad m_{B}^{\prime}=m_{B, 0}^{\prime}+m_{B, \pi}^{\prime},
$$

where the second index stands for the energy. We are looking for the topological invariants of the bulk parts of the walk, $v_{L, \varepsilon}$, and $v_{R, \varepsilon}$, where $\varepsilon=0, \pi$, whose differences give us the number of topologically protected edge states separately for each energy,

$$
v_{L, \varepsilon}-v_{R, \varepsilon}=m_{A, \varepsilon}^{\prime}-m_{B, \varepsilon}^{\prime} .
$$

The first step towards the topological invariants is the standard winding number $v^{\prime 3}$ associated to the bulk effective Hamiltonian $H_{\text {eff }}^{\prime}$, in the time frame where the Floquet operator is $U^{\prime}$. This is obtained from the bulk, translational invariant part of the Floquet operator, diagonal in momentum space: $U^{\prime}=\sum_{k}|k\rangle\langle k| \otimes U^{\prime}(k)$, and $U^{\prime}(k)=e^{-i H_{\text {eff }}(k)}$. Instead of the effective Hamiltonian, it is convenient to calculate with $H^{\prime}(k)=\sin \left[H_{\text {eff }}(k)\right]$. This has the same CS and the same winding number as $H_{\text {eff }}(k)$ (and as its flattened version $\left.Q=\operatorname{sgn}\left[H_{\mathrm{eff}}(k)\right]^{3}\right)$, but can be obtained much more efficiently via $H^{\prime}(k)=\left[U^{\prime}(k)^{\dagger}-U^{\prime}(k)\right] /(2 i)$. In a basis where $\Gamma=$ $\operatorname{diag}(1, \ldots, 1,-1, \ldots,-1)$ is a diagonal matrix with an equal number of +1 and -1 elements, the matrix of $H^{\prime}(k)$ is block off diagonal because of CS. We name its upper right block 
$h^{\prime}(k)$. The winding number $v^{\prime}$ reads

$$
v^{\prime}=\frac{1}{2 \pi i} \int_{-\pi}^{\pi} d k \frac{d}{d k} \ln \operatorname{det} h^{\prime}(k) .
$$

The winding number $v^{\prime}$ is related to the difference of the bulk polarizations on the two sublattices in bulk. Therefore it cannot differentiate between 0 and $\pi$ energy edge states, and can only be used to obtain the sum of all topologically protected edge states around $x \approx d$ :

$$
v_{L}^{\prime}-v_{R}^{\prime}=m_{A, 0}^{\prime}+m_{A, \pi}^{\prime}-m_{B, 0}^{\prime}-m_{B, \pi}^{\prime} .
$$

However, there is the other CS time frame, $U^{\prime \prime}$, where we have

$$
v_{L}^{\prime \prime}-v_{R}^{\prime \prime}=m_{A, 0}^{\prime \prime}+m_{A, \pi}^{\prime \prime}-m_{B, 0}^{\prime \prime}-m_{B, \pi}^{\prime \prime} .
$$

We need to combine the information from the two CS time frames to obtain the topological invariants.

We can obtain a simple connection between the two CS time frames by considering an edge state. In the time frame of $U^{\prime}=F G$, the edge state has a wave function $\Psi$, entirely on sublattice $A$ (or $B$ ), i.e., $\Gamma \Psi=(-1)^{g} \Psi$, with $g=0$ (or 1). In other time frames, where $U_{T}$ has no $\mathrm{CS}$, the energy of the edge state has to remain the same, but its wave function can extend over both sublattices. In the other CS time frame $U^{\prime \prime}=G F$, however, its wave function, $\Phi=G \Psi$, again has to to be entirely on a single sublattice. This can be $A$ (or $B$ ), whereby $\Gamma \Phi=(-1)^{f} \Phi$, with $f=0$ (or 1). Consider $G F \Phi=G \Gamma G^{-1} \Gamma \Phi=G \Gamma G^{-1}(-1)^{f} \Phi=$ $G \Gamma(-1)^{f} \Psi=(-1)^{g+f} G \Psi=(-1)^{g+f} \Phi$. This shows that 0 $(\pi)$ energy edge states are on the same (opposite) sublattice in the two CS time frames. This can be summarized as

$$
m_{A}^{\prime \prime}=m_{A, 0}^{\prime}+m_{B, \pi}^{\prime} ; \quad m_{B}^{\prime \prime}=m_{B, 0}^{\prime}+m_{A, \pi}^{\prime} .
$$

To obtain the number of protected edge states at zero and $\pi$ energies separately, we substitute Eq. (20) into Eqs. (18), (15), and (19), and rearrange to obtain

$$
\begin{aligned}
& m_{A, 0}^{\prime}-m_{B, 0}^{\prime}=\frac{v_{L}^{\prime}+v_{L}^{\prime \prime}}{2}-\frac{v_{R}^{\prime}+v_{R}^{\prime \prime}}{2}, \\
& m_{A, \pi}^{\prime}-m_{B, \pi}^{\prime}=\frac{v_{L}^{\prime}-v_{L}^{\prime \prime}}{2}-\frac{v_{R}^{\prime}-v_{R}^{\prime \prime}}{2} .
\end{aligned}
$$

We compare this with Eq. (16), and read off the bulk topological invariants $\left(v_{0}, v_{\pi}\right)$ as

$$
\left(v_{0}, v_{\pi}\right)=\left(\frac{v^{\prime}+v^{\prime \prime}}{2}, \frac{v^{\prime}-v^{\prime \prime}}{2}\right) .
$$

This, the bulk-edge correspondence for DTQW's with CS, is the main result of this Rapid Communication.

Having derived a general formula for the topological invariant of 1D DTQWs with CS, we now discuss an example where the differences between CS and PHS come into play. To arrive to the example, first consider the "split-step walk" of Kitagawa et al., ${ }^{9}$ given by $U_{0}=S_{+} R(0, \phi) S_{-} R\left(0, \theta_{1}\right)$. There, both PHS and CS are present, and we find that the $v_{\varepsilon}$ are one-to-one functions of the invariants $Q_{\varepsilon}$ induced by PHS: ${ }^{13}$ $\nu_{\varepsilon}=1 / 2-Q_{\varepsilon}$, for both $\varepsilon=0, \pi$. (An interesting special case is the simple quantum walk, obtained by setting $\phi=0$.) We can break PHS by using nonzero angles $\chi$. To be able to break CS, we consider a longer period of pulses, a "4-step DTQW,"

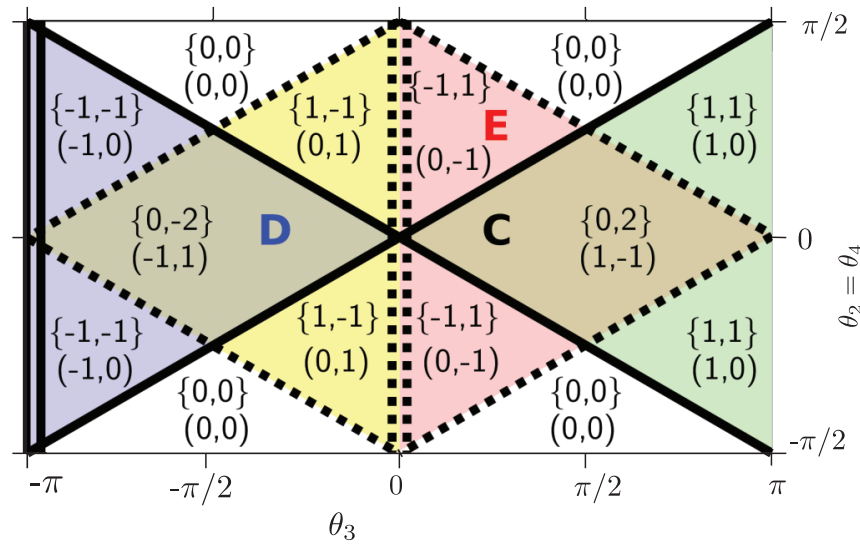

FIG. 2. (Color online) Parameter space of the 4-step DTQW with PHS ensured by $\chi_{j}=0$, CS ensured by $\theta_{2}=\theta_{4}$, and $\theta_{1}$ set to 0 . The DTQW has effective Hamiltonians with gaps around both $\varepsilon=0$ and $\varepsilon=\pi$, except at the gapless points where gaps close at $\varepsilon=0$ (solid lines) or $\varepsilon=\pi$ (dashed lines). Single lines indicate that the gap closes at a single $k$, at either $k=0$ or $k=\pi$. Double lines indicate double gap closings, at $k= \pm \pi / 2$. For each gapped domain, the corresponding pair of winding numbers $\left\{v^{\prime}, v^{\prime \prime}\right\}$ as well as the pair of topological invariants $\left(v_{0}, v_{\pi}\right)$, cf. Eq. (23), are shown. Letters "C", "D," and "E" indicate sets of parameters used for the inhomogeneous quantum walk, with rotation as in Eq. (25).

given by

$$
U_{0}=S_{+} R_{4} S_{+} R_{3} S_{-} R_{2} S_{-} R_{1} .
$$

This walk has no CS if $R_{2} \neq R_{4}$, but has CS if $R_{2}=R_{4}$, with $F=R_{3}^{1 / 2} S_{-} R_{2} S_{-} R_{1}^{1 / 2}$ and $G=R_{1}^{1 / 2} S_{+} R_{4} S_{+} R_{3}^{1 / 2}$. The 4-step walk also has the advantage that the effective Hamiltonian will have longer range hoppings, and thus we can expect higher values of the winding numbers. This is entirely analogous to adding a third nearest-neighbor hopping term to the SSH model.

The topological invariants in a section of the phase space of the 4-step DTQW with both CS and PHS (Cartan class $\mathrm{BDI}^{3}$ ) are shown in Fig. 2. Here, we set all $\chi_{j}=0$ to ensure PHS, $\theta_{2}=\theta_{4}$ to ensure CS, and set $\theta_{1}=0$ for simplicity. We restrict $\theta_{2}$ to $-\pi / 2<\theta_{2}<\pi / 2$ since adding $\pi$ to both $\theta_{2}$ and $\theta_{4}$ just brings two factors of -1 that cancel out in both time frames with CS. Generic values of $\theta_{2}=\theta_{4}$ and $\theta_{3}$ give effective Hamiltonians with gaps around both $\varepsilon=0$ and $\varepsilon=\pi$. Examples for these are the points $C\left(\theta_{2}=\pi / 20, \theta_{3}=\pi / 4\right), D$ $\left(\theta_{2}=0, \theta_{3}=-\pi / 4\right)$, and $E\left(\theta_{2}=\pi / 4, \theta_{3}=\pi / 4\right)$.

To see the effects of breaking the symmetries on edge states, we consider two inhomogeneous systems, consisting of two domains of 40 sites each, with sharp boundaries in between. The inhomogeneous rotations read

$$
R_{j}=\sum_{x=1}^{40}|x\rangle\left\langle x\left|\otimes R_{j, X}+\sum_{x=41}^{80}\right| x\right\rangle\langle x| \otimes R_{j, C},
$$

where $X=D$ or $X=E$, and $C$ refer to the parameter sets of defined in the previous paragraph and indicated in Fig. 2.

We break PHS (realizing Cartan class AIII) in a controlled way by introducing a nonzero $\chi_{3}$ in the bulk $0<x<41$. As long as the bulk gaps are still open, breaking PHS does not change the edge state energies, as shown in Figs. 3(A1) 

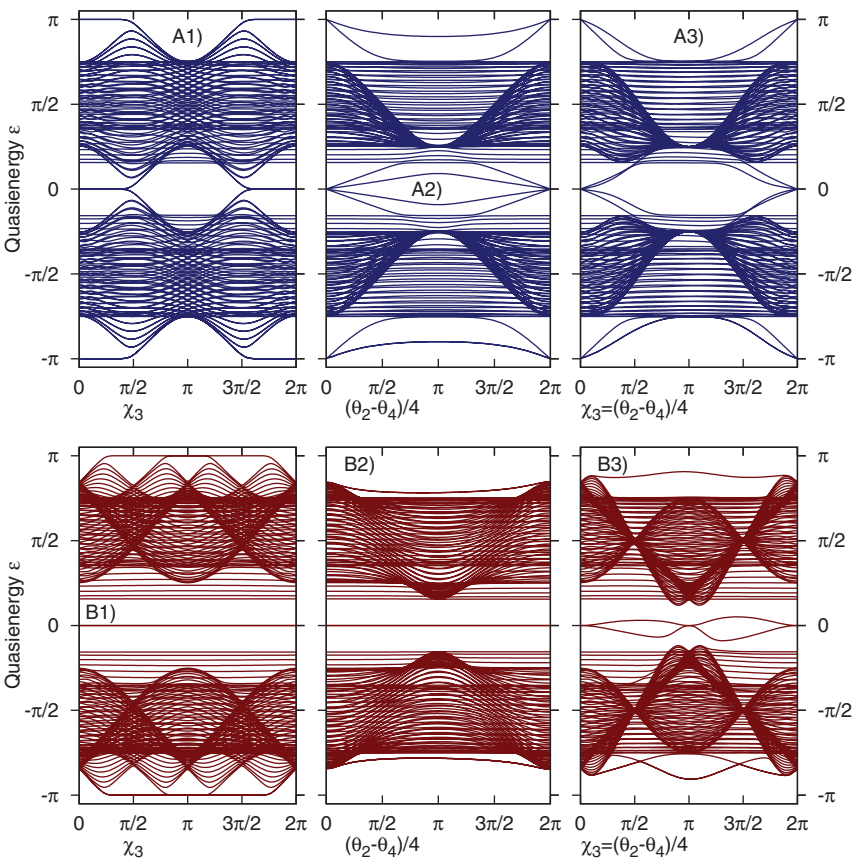

FIG. 3. (Color online) Spectra of an inhomogeneous "4-step walk" on $N=60$ sites as defined by Eqs. (24) and (25), with two domains: D and C (top row), and $\mathrm{E}$ and $\mathrm{C}$ (bottom row). (Left) We break PHS via $\chi_{3}$ for $x<41$ on (A1) and (B1). As long as the bulk gaps are still open, the edge state energies do not change as they are still protected by CS. (Middle) (A2) and (B2): we break CS by setting $\theta_{2} \rightarrow \theta_{2}+\Delta \theta, \theta_{4} \rightarrow \theta_{4}-\Delta \theta$ for $n<31$. This lifts the degeneracy of edge states on the same edge pairwise. At the interface between $\mathrm{E}$ and $\mathrm{C}$, the unpaired edge state remains (B2). (Right) (A3) and (B3): as both PHS and CS are broken, no topologically protected edge states remain. In (B3), the unpaired edge states at both edges are displaced in energy.

and 3(B1). The edge-state energies are still protected by CS, and can only move from their original values if the bulk gap closes (at $\chi_{3}=\pi / 2$ for the D-C boundary). We break CS (realizing Cartan class D), by changing $\theta_{2}-\theta_{4}$ in the " $L$ " bulk. A pair of edge states on the same edge at the same energy can now break apart, becoming PHS partners of each other. This can be seen in Fig. 3(A2) at both 0 and $\pi$ energy. However, a single edge state, as the one between bulks $B$ and $C$, is still protected by PHS when CS is broken, as seen in Fig. 3(B2). Finally, to check that no extra hidden symmetries remain, we break both $\mathrm{CS}$ and PHS (realizing Cartan class A). In that case, the edge-state energies are not protected anymore, cf Figs. 3(A3) and 3(B3). This shows that our description of the relevant symmetries of the DTQW was indeed exhaustive.

To summarize, we gave a definition of CS for DTQWs and derived the corresponding bulk topological invariants, using the fact that the walk is defined by a sequence of operations, rather than just by its unitary time-step operator. The "timeshifting" approach presented here based on finding the "CS time frames" should generalize to periodically driven quantum systems. ${ }^{15-19}$ In such setups, PHS has been shown to lead to two types of "Floquet Majorana fermions," 12 which should have clear signals in transport ${ }^{20}$ and can also be useful for quantum information processing. ${ }^{21}$ Theoretical proposals have already seen several such states at a single edge if the driving also ensures $\mathrm{CS}^{22}$ The bulk topological invariant controlling the number of these edge states is as yet unknown, but it could be derived using the approach of this Rapid Communication.

Although two-dimensional DTQWs have already been realized in experiments, ${ }^{23}$ their topological invariants are largely unexplored. In two dimensions, edge states can exist in the absence of symmetries; the related bulk-boundary correspondence for periodically driven systems has only recently been found. ${ }^{24}$ The approach of identifying the "symmetric time frames" could be a key idea for the description of other symmetry classes for both periodically driven quantum systems and DTQWs.

JKA thanks J. Edge and A. Gábris, and HO thanks N. Kawakami, Y. Nishimura, and T. Kitagawa for helpful discussions. This research was realized in the frames of TAMOP 4.2.4. A/1-11-1-2012-0001 "National Excellence Program elaborating and operating an inland student and researcher personal support system," subsidized by the European Union and co-financed by the European Social Fund. This work was also supported by the Hungarian National Office for Research and Technology under the contract ERC_HU_09 OPTOMECH and the Hungarian Academy of Sciences (Lendület Program, LP2011-016). H. O. was supported by Grant-in-Aid (Nos. 25800213 and 25390113) from the Japan Society for Promotion of Science.
${ }^{1}$ M. Z. Hasan and C. L. Kane, Rev. Mod. Phys. 82, 3045 (2010).

${ }^{2}$ X.-L. Qi and S.-C. Zhang, Rev. Mod. Phys. 83, 1057 (2011).

${ }^{3}$ S. Ryu, A. P. Schnyder, A. Furusaki, and A. W. W. Ludwig, New J. Phys. 12, 065010 (2010).

${ }^{4}$ P. Hauke, O. Tieleman, A. Celi, C. Ölschläger, J. Simonet, J. Struck, M. Weinberg, P. Windpassinger, K. Sengstock, M. Lewenstein, et al., Phys. Rev. Lett. 109, 145301 (2012).

${ }^{5}$ Y. Aharonov, L. Davidovich, and N. Zagury, Phys. Rev. A 48, 1687 (1993).

${ }^{6}$ J. Kempe, Contemp. Phys. 44, 307 (2003).

${ }^{7}$ A. Ambainis, Int. J. Quantum Inf. 01, 507 (2003).

${ }^{8}$ A. Schreiber, K. N. Cassemiro, V. Potoček, A. Gábris, P. J. Mosley, E. Andersson, I. Jex, and C. Silberhorn, Phys. Rev. Lett. 104, 050502 (2010).
${ }^{9}$ T. Kitagawa, M. S. Rudner, E. Berg, and E. Demler, Phys. Rev. A 82, 033429 (2010).

${ }^{10}$ T. Kitagawa, Quantum Inf. Process. 11, 1107 (2012).

${ }^{11}$ H. Obuse and N. Kawakami, Phys. Rev. B 84, 195139 (2011).

${ }^{12}$ L. Jiang, T. Kitagawa, J. Alicea, A. R. Akhmerov, D. Pekker, G. Refael, J. I. Cirac, E. Demler, M. D. Lukin, and P. Zoller, Phys. Rev. Lett. 106, 220402 (2011).

${ }^{13}$ J. K. Asbóth, Phys. Rev. B 86, 195414 (2012).

${ }^{14}$ T. Kitagawa, M. A. Broome, A. Fedrizzi, M. S. Rudner, E. Berg, I. Kassal, A. Aspuru-Guzik, E. Demler, and A. G. White, Nat. Commun. 3, 882 (2012).

${ }^{15}$ T. Kitagawa, E. Berg, M. Rudner, and E. Demler, Phys. Rev. B 82, 235114 (2010). 
${ }^{16}$ N. H. Lindner, G. Refael, and V. Galitski, Nat. Phys. 7, 490 (2011).

${ }^{17}$ J. Cayssol, B. Dóra, F. Simon, and R. Moessner, Phys. Status Solidi RRL 7, 101 (2013).

${ }^{18}$ K. Sun, W. V. Liu, A. Hemmerich, and S. D. Sarma, Nat. Phys. 8, 6770 (2012).

${ }^{19}$ J. P. Dahlhaus, J. M. Edge, J. Tworzydło, and C. W. J. Beenakker, Phys. Rev. B 84, 115133 (2011).

${ }^{20}$ A. Kundu and B. Seradjeh, Phys. Rev. Lett. (in press), arXiv:1301.4433.
${ }^{21}$ D. E. Liu, A. Levchenko, and H. U. Baranger, Phys. Rev. Lett. 111, 047002 (2013).

${ }^{22}$ Q.-J. Tong, J.-H. An, J. Gong, H.-G. Luo, and C. H. Oh, Phys. Rev. B 87, 201109 (2013).

${ }^{23}$ A. Schreiber, A. Gábris, P. P. Rohde, K. Laiho, M. Štefaňák, V. Potoček, C. Hamilton, I. Jex, and C. Silberhorn, Science 336, 55 (2012).

${ }^{24}$ M. S. Rudner, N. H. Lindner, E. Berg, and M. Levin, Phys. Rev. X 3, 031005 (2013). 